\title{
Iterative Approximations for Zeros of Sum of Accretive Operators in Banach Spaces
}

\author{
Yekini Shehu \\ Department of Mathematics, University of Nigeria, Nsukka, Nigeria \\ Correspondence should be addressed to Yekini Shehu; yekini.shehu@unn.edu.ng \\ Received 27 October 2015; Revised 13 December 2015; Accepted 24 December 2015 \\ Academic Editor: Adrian Petrusel
}

Copyright (c) 2016 Yekini Shehu. This is an open access article distributed under the Creative Commons Attribution License, which permits unrestricted use, distribution, and reproduction in any medium, provided the original work is properly cited.

We study the approximation of zero for sum of accretive operators using a modified Mann type forward-backward splitting algorithm and obtain strong convergence of the sequence generated by our scheme to the zero of sum of accretive operators in uniformly convex real Banach spaces which are also uniformly smooth. Our result is new and complements many recent and important results in this direction in the literature.

\section{Introduction and Preliminaries}

Let $E$ be a real Banach space. The modulus of convexity $\delta_{E}$ : $[0,2] \rightarrow[0,1]$ is defined as

$$
\begin{aligned}
& \delta_{E}(\epsilon) \\
& \quad=\inf \left\{1-\frac{\|x+y\|}{2}:\|x\|=1=\|y\|,\|x-y\| \geq \epsilon\right\} .
\end{aligned}
$$

$E$ is called uniformly convex if $\delta_{E}(\epsilon)>0$ for any $\epsilon \in(0,2]$ and $p$-uniformly convex if there is $c_{p}>0$ so that $\delta_{E}(\epsilon) \geq c_{p} \epsilon^{p}$ for any $\epsilon \in(0,2]$. The modulus of smoothness $\rho_{E}(\tau):[0, \infty) \rightarrow$ $[0, \infty)$ is defined by

$$
\rho_{E}(\tau)=\left\{\frac{\|x+\tau y\|+\|x-\tau y\|}{2}-1:\|x\|=\|y\|=1\right\} .
$$

$E$ is called uniformly smooth if $\lim _{n \rightarrow \infty}\left(\rho_{E}(\tau) / \tau\right)=0$ and $q$ uniformly smooth if there is $C_{q}>0$ so that $\rho_{E}(\tau) \leq C_{q} \tau^{q}$ for any $\tau>0$. Hilbert spaces, $L_{p}$ (or $\ell_{p}$ ) spaces, $1<p<\infty$, and the Sobolev spaces, $W_{m}^{p}, 1<p<\infty$, are $q$-uniformly smooth. Hilbert spaces are 2-uniformly smooth while

$$
\begin{aligned}
& L_{p}\left(\text { or } \ell_{p}\right) \\
& \text { or } W_{m}^{p} \text { is } \begin{cases}p \text {-uniformly smooth } & \text { if } 1<p \leq 2 \\
2 \text {-uniformly smooth } & \text { if } p \geq 2 .\end{cases}
\end{aligned}
$$

It is shown in [1] that there is no Banach space which is $q$ uniformly smooth with $q>2$. It is obvious that $q$-uniformly smooth Banach space must be uniformly smooth. by

The normalized duality mapping $J: E \rightarrow 2^{E^{*}}$ is defined

$$
J x:=\left\{f \in E^{*}:\langle x, f\rangle=\|x\|^{2}=\|f\|^{2}\right\}, \quad x \in E .
$$

It is well known that $J$ is single-valued and norm-to-norm uniformly continuous on each bounded subsets of $E$ if $E$ is a real smooth and uniformly convex Banach space (please see [2]). In the sequel, we will denote by $j$ the single-valued normalized duality mapping. If $E$ is reduced to the Hilbert space $H$, then $J \equiv I$ is the identity mapping.

Let $T: E \rightarrow E$ be a mapping on $E$. We will use $F(T)$ to denote its set of fixed points; that is, $F(T):=\{x \in E: T x=x\}$. Furthermore, we say that $T$ is a fixed contraction if there exists a constant $k \in(0,1)$ such that, for all $x, y \in E$, we have $\| T x-$ $T y\|\leq k\| x-y \|$. The mapping $T$ is said to be nonexpansive if $k=1$. Thus, $T$ is nonexpansive if $\|T x-T y\| \leq\|x-y\|$.

Let $C$ be a nonempty, closed, and convex subset of $E$ and let $Q$ be a mapping of $E$ onto $C$. Then $Q$ is said to be sunny [3] if $Q(Q(x)+t(x-V Q(x)))=Q(x)$, for all $x \in E$ and $t \geq 0$. A mapping $Q$ of $E$ into $E$ is said to be a retraction [3] if $Q^{2}=Q$. If a mapping $Q$ is a retraction, then $Q(z)=z$ for every $z \in R(Q)$, where $R(Q)$ is the range of $Q$. In [4], it was shown that if $E$ is uniformly smooth and if $D$ is the fixed point 
set of a nonexpansive mapping from $C$ into itself, then there is a unique sunny nonexpansive retraction from $C$ onto $D$. We know that, in a uniformly smooth Banach space, a retraction $Q: C \rightarrow D$ is sunny and nonexpansive, if and only if

$$
\langle x-Q(x), j z-Q(x)\rangle \leq 0, \quad \forall x \in C, z \in D \text {. }
$$

A set-valued operator $A: E \rightarrow 2^{E}$, with domain $D(A)$ and range $R(A)$, is said to be accretive if, for all $t>0$ and every $x, y \in D(A)$,

$$
\|x-y\| \leq\|x-y+t(u-v)\|, \quad u \in A x, v \in A y .
$$

Equivalently, by Lemma 1.1 of Kato [5] we know that $A$ is accretive if and only if, for each $x, y \in D(A)$, there exists $j(x-$ $y) \in J(x-y)$ such that

$$
\langle u-v, j(x-y)\rangle \geq 0, \quad u \in A x, v \in A y .
$$

Furthermore, an accretive operator $A$ is said to be $m$-accretive if the range $R(I+\lambda A)=E$ for all $\lambda>0$. Given $\alpha>0$ and $q \in(1, \infty)$, we say that an accretive operator $A$ is $\alpha$-inverse strongly accretive ( $\alpha$-isa) of order $q$ if, for each $x, y \in D(A)$, there exists $j_{q}(x-y) \in J_{q}(x-y)$ such that

$$
\left\langle u-v, j_{q}(x-y)\right\rangle \geq \alpha\|u-v\|^{q}, \quad u \in A x, v \in A y .
$$

When $q=2$, we simply say $\alpha$-isa, instead of $\alpha$-isa of order 2; that is, $A$ is $\alpha$-isa if, for each $x, y \in D(A)$, there exists $j(x-y) \in$ $J(x-y)$ such that

$$
\langle u-v, j(x-y)\rangle \geq \alpha\|u-v\|^{2}, \quad u \in A x, v \in A y .
$$

Let $E$ be a real uniformly convex Banach space which is also uniformly smooth. Suppose $A: E \rightarrow 2^{E}$ is an $m$-accretive operator and $B: E \rightarrow E$ an $\alpha$-inverse strongly accretive. In this paper, we will consider the following inclusion problem (assuming that the solution exists): find $x \in E$ such that

$$
0 \in A x+B x
$$

Problem (10) is a very general format for certain concrete problems in machine learning, image processing and linear inverse problem, and many nonlinear problems such as convex programming, variational inequalities, split feasibility problem, and minimization problem.

It is well known that a forward-backward splitting method (please see, e.g., [6-9]) is the classical method for solving problem (10). This forward-backward splitting method is given by $x_{1} \in E$ and

$$
x_{n+1}=(I+r A)^{-1}\left(x_{n}-r B x_{n}\right), \quad n \geq 1,
$$

where $r>0$. This method generalizes the proximal point algorithm (please see [10-14]) and the gradient method (see, e.g., [15]). There have been many works concerning the problem of finding zero points of the sum of two monotone operators (in Hilbert spaces) and accretive operators (in Banach spaces). For more details, please, see [16-20] and the references contained therein. Motivated by the results of López et al. [21], Cholamjiak [22] and Wei and Shi [23] have recently extended the results of López et al. [21] from Halpern-type forward-backward method to viscositytype forward-backward method with fixed contraction in $q$ uniformly smooth Banach spaces which are also uniformly convex.

Remark 1. We note that for $q=2$ the class of inverse strongly accretive of order $q$ coincides with that of inverse strongly accretive. Thus, in the case of $q \neq 2$, (8) is not equivalent to (9). Furthermore, for $q<2$, inverse strongly accretive operators of order $q$ do represent a subclass of inverse strongly accretive operators (see [24]). In light of above comments, the class of inverse strongly accretive operators of order $q$ considered in the results of Cholamjiak [22], López et al. [21], and Wei and Shi [23] is a subclass of inverse strongly accretive operators we will consider in this paper.

Recently, Wei and Duan [25] studied the problem of finding zeros of the sum of finitely many $m$-accretive operators and finitely many $\alpha$-inversely strongly accretive operators in a real smooth and uniformly convex Banach space (i.e., find $x \in E$ such that $\left.0 \in \sum_{i=1}^{N}\left(A_{i} x+B_{i} x\right)\right)$. They proved the following strong convergence result.

Theorem 2. Let $E$ be a real smooth and uniformly convex Banach space and let $C$ be a nonempty, closed, and convex sunny nonexpansive retraction of $E$, and let $Q_{C}$ be the sunny nonexpansive retraction of $E$ onto $C$. Let $f: E \rightarrow E$ be a fixed contractive mapping with coefficient $k \in(0,1)$, and let $T$ : $E \rightarrow E$ be a strongly positive linear bounded operator with coefficient $\bar{\gamma}$. Suppose that the duality mapping $J: E \rightarrow E^{*}$ is weakly sequentially continuous at zero, and $0<\eta<\bar{\gamma} / 2 k$. Let $A_{i}: C \rightarrow 2^{E}$ be $m$-accretive operator and let $B_{i}: C \rightarrow E$ be $\alpha$-inversely strongly accretive operator, where $i=1,2, \ldots, N$. Suppose that, for $r>0$ and $i=1,2, \ldots, N$,

$$
\left\langle B_{i} x-B_{i} y, J\left(\left(I-r B_{i}\right) x-\left(I-r B_{i}\right)\right) y\right\rangle \geq 0 .
$$

Let $\left\{x_{n}\right\}$ be generated by

$$
\begin{aligned}
x_{0} \in C & \\
y_{n}= & Q_{C}\left[\left(1-\alpha_{n}\right)\left(x_{n}+e_{n}\right)\right], \\
z_{n}= & \left(1-\beta_{n}\right) x_{n} \\
& +\beta_{n}\left[a_{0} y_{n}+\sum_{i=1}^{N} a_{i} J_{r_{n, i}}^{A_{i}}\left(y_{n}-r_{n, i} B_{i} y_{n}\right)\right], \\
x_{n+1}= & \gamma_{n} \eta f\left(x_{n}\right)+\left(I-\gamma_{n} T\right) z_{n}, \quad n \geq 0,
\end{aligned}
$$

$0<a_{m}<1$, for $m=0,1, \ldots, N, \sum_{m=0}^{N} a_{m}=1$. Suppose $\left\{e_{n}\right\} \subset$ $E,\left\{\alpha_{n}\right\},\left\{\beta_{n}\right\}$, and $\left\{\gamma_{n}\right\}$ are three sequences in $(0,1)$ and $\left\{r_{n, i}\right\} \subset$ $(0,+\infty)$ satisfying the following conditions:

(i) $\sum_{n=0}^{\infty} \gamma_{n}=\infty, \gamma_{n} \rightarrow 0,\left(\gamma_{n-1}\right) / \gamma_{n} \rightarrow 0, \beta_{n} \rightarrow 1$, $\alpha_{n} \rightarrow 0$, as $n \rightarrow \infty$

(ii) $\sum_{n=0}^{\infty}\left|\alpha_{n+1}-\alpha_{n}\right|<+\infty, \sum_{n=0}^{\infty}\left|\beta_{n+1}-\beta_{n}\right|<+\infty$, $\sum_{n=0}^{\infty}\left(1-\gamma_{n} \bar{\gamma}\right) \alpha_{n} \beta_{n}<+\infty$ 
(iii) $\sum_{n=0}^{\infty}\left|\alpha_{n+1, i}-\alpha_{n, i}\right|<+\infty$ and $r_{n, i} \geq \epsilon>0$, for $n \geq 0$ and $i=1,2, \ldots, N$;

(iv) $\sum_{n=0}^{\infty}\left\|e_{n}\right\|<\infty$.

If $\sum_{i=1}^{N}\left(A_{i}+B_{i}\right)^{-1}(0) \neq \emptyset$, then $\left\{x_{n}\right\}$ converges strongly to a point $p_{0} \in \sum_{i=1}^{N}\left(A_{i}+B_{i}\right)^{-1}(0)$, which is the unique solution of the following variational inequality: for all $z \in \sum_{i=1}^{N}\left(A_{i}+\right.$ $\left.B_{i}\right)^{-1}(0)$,

$$
\left\langle(T-\eta f) p_{0}, J\left(p_{0}-z\right)\right\rangle \leq 0 .
$$

We observe that Theorem 2 extends the results of López et al. [21] from $q$-uniformly smooth Banach spaces which are also uniformly convex to uniformly smooth and uniformly convex Banach spaces under the condition that the normalized duality mapping $J$ is weakly sequentially continuous at zero. We also observe that this result is not applicable in $L_{p}$, $p \neq 2$. This leads to this natural question.

Question 1. Can we obtain strong convergence result for approximation of zero of sum of accretive operators in uniformly smooth Banach spaces which are also uniformly convex without assuming that the normalized duality mapping $J$ is weakly sequentially continuous at zero or $\langle B x-B y, J((I-$ $r B) x-(I-r B)) y>\geq 0$ ?

Our interest in this paper is to answer the above question in the affirmative. That is, we establish strong convergence result for approximation of zero of sum of accretive operators in uniformly smooth Banach spaces which are also uniformly convex without assuming that the normalized duality mapping $J$ is weakly sequentially continuous at zero or $\langle B x-B y, J((I-r B) x-(I-r B)) y\rangle \geq 0$. To achieve this, apply generalized forward-backward method which involves viscosity approximation method with fixed contraction and weakly contractive mappings.

In the sequel, we will assume that $A$ is an $m$-accretive operator. Then we can define, for each $r>0$, the resolvent $J_{r}^{A}: R(I+r A) \rightarrow D(A)$ by $J_{r}^{A}:=(I+r A)^{-1}$, and the Yosida approximation $A_{r}: R(I+r A) \rightarrow R(A)$ by $A_{r}=\left(I-J_{r}^{A}\right) / r$. Denote by $A^{-1}(0)$ the zero set of $A$; that is, $A^{-1}(0):=\{x \in$ $D(A): 0 \in A x\}$. It is well known that $J_{r}^{A}$ is single-valued and nonexpansive, $F\left(J_{r}^{A}\right)=A^{-1}(0)$ and $A_{r} x \in A\left(J_{r}^{A} x\right)$ for every $x \in R(I+r A)$, where $F\left(J_{r}^{A}\right):=\left\{x \in R(I+r A): J_{r}^{A} x=x\right\}$.

In what follows we will make use of the following lemmas.

Lemma 3 (see Maingé [26]). Let $\left\{s_{n}\right\}$ be a real sequence that does not decrease at infinity, in the sense that there exists a subsequence $\left\{s_{n_{k}}\right\}$ so that $s_{n_{k}} \leq s_{n_{k}+1}$ for all $k \geq 1$.

For every $n>n_{0}$ define an integer sequence $\left\{s_{n_{k}}\right\}$ as

$$
\tau_{(n)}=\max \left\{n_{0} \leq k \leq n: s_{k}<s_{k+1}\right\}
$$

Then $\tau_{(n)} \rightarrow \infty$ as $n \rightarrow \infty$ and for all $n>n_{0}$

$$
\max \left\{s_{\tau_{(n)}}, s_{n}\right\} \leq s_{\tau_{(n)}+1}
$$

Lemma 4 (see Xu [27]). Assume $\left\{a_{n}\right\}$ is a sequence of nonnegative real numbers such that

$$
a_{n+1} \leq\left(1-\gamma_{n}\right) a_{n}+\gamma_{n} \delta_{n}, \quad n \geq 0,
$$

where $\left\{\gamma_{n}\right\}$ is a sequence in $(0,1)$ and $\left\{\delta_{n}\right\}$ is a sequence in $\mathbb{R}$ such that

(i) $\sum_{n=0}^{\infty} \gamma_{n}=\infty$,

(ii) $\lim \sup _{n \rightarrow \infty} \delta_{n} \leq 0$ or $\sum_{n=0}^{\infty}\left|\delta_{n} \gamma_{n}\right|<\infty$.

Then $\lim _{n \rightarrow \infty} a_{n}=0$.

Lemma 5 (see $\mathrm{Xu}$ [28]). Let $C$ be a nonempty closed convex subset of a uniformly smooth Banach space $E$ and let $T: C \rightarrow$ $C$ be a nonexpansive mapping with a fixed point. Let $f: E \rightarrow$ $E$ be a fixed contraction with coefficient $k \in(0,1)$. If there exists a bounded sequence $\left\{x_{n}\right\}$ such that $\lim _{n \rightarrow \infty}\left\|x_{n}-T x_{n}\right\|=0$ and $p=\lim _{t \rightarrow 0} z_{t}$ exists, where $\left\{z_{t}\right\}$ is defined by $z_{t}=t f\left(z_{t}\right)+(1-$ t) $T z_{t}$ and $p$ solves the variational inequality

$$
\langle(I-f) p, j(p-x)\rangle \leq 0, \quad \forall x \in F(T),
$$

then

$$
\limsup _{n \rightarrow \infty}\left\langle f(p)-p, j\left(x_{n}-p\right)\right\rangle \leq 0 .
$$

Lemma 6. Let $E$ be a real Banach space. Then, for all $x, y \in E$, one has

$$
\|x+y\|^{2} \leq\|x\|^{2}+2\langle y, j(x+y)\rangle .
$$

Lemma 7 (see López et al. [21]). Let $E$ be a real Banach space. Let $A: E \rightarrow 2^{E}$ be an $m$-accretive operator and let $B: E \rightarrow E$ be an $\alpha$-inverse strongly accretive mapping on $E$. Then one has

(i) for $r>0, F\left(T_{r}\right)=(A+B)^{-1}(0)$,

(ii) for $0<s \leq r$ and $x \in E$, $\left\|x-T_{s} x\right\| \leq 2\left\|x-T_{r} x\right\|$.

Lemma 8 (see Suantai and Cholamjiak [29]). Let E be a real Banach space with Fréchet differentiable norm. For $x \in E$, let $\beta^{*}(t)$ be defined for $0<t<\infty$ by

$$
\begin{aligned}
& \beta^{*}(t) \\
& =\sup \left\{\left|\frac{\|x+t y\|^{2}-\|x\|^{2}}{t}-2\langle y, j(x)\rangle\right|:\|y\|=1\right\} .
\end{aligned}
$$

Then, $\lim _{t \rightarrow 0^{+}} \beta^{*}(t)=0$ and

$$
\|x+h\|^{2} \leq\|x\|^{2}+2\langle h, j(x)\rangle+\|h\| \beta^{*}(\|h\|)
$$

for all $h \in E \backslash\{0\}$.

Remark 9. In a real Hilbert space, we see that $\beta^{*}(t)=t$ for $t>0$.

In the result of Suantai and Cholamjiak [29], the authors assumed that $\beta^{*}(t) \leq 2 t$ for $t>0$. This naturally leads to this important question. 
Question 2. What uniformly smooth Banach spaces (except Hilbert spaces) satisfy the assumption $\beta^{*}(t) \leq 2 t$ for $t>0$ ? In particular, do $L_{p}$ spaces, $1<p<\infty$, satisfy it?

In $E=L_{p}, 2 \leq p<\infty$, we know that

$$
\begin{array}{r}
\|x+y\|^{2} \leq\|x\|^{2}+2\langle y, j(x)\rangle+(p-1)\|y\|^{2}, \\
\forall x, y \in E .
\end{array}
$$

Then $\beta^{*}$ in $(21)$ is estimated by $\beta^{*}(t) \leq(p-1) t$ for $t>0$.

In our more general setting, throughout this paper, we will assume that

$$
\beta^{*}(t) \leq c t, \quad t>0 \text { and for some } c>1,
$$

where $\beta^{*}$ is the function appearing in (21).

By following the same method of proof as contained in the proof of Lemma 3.3 of [21], we have the following lemma.

Lemma 10. Let $E$ be a real uniformly convex with Fréchet differentiable norm. Assume that $B$ is a single-valued $\alpha$-inverse strongly accretive mapping on $E$. Then, given $s>0$, there exists a continuous, strictly increasing and convex function $\varphi: \mathbb{R}^{+} \rightarrow$ $\mathbb{R}^{+}$with $\varphi(0)=0$ such that, for all $x, y \in E$,

$$
\begin{aligned}
& \left\|T_{r} x-T_{r} y\right\|^{2} \\
& \leq\|x-y\|^{2}-r(2 \alpha-r c)\|B x-B y\|^{2} \\
& \quad-\varphi\left(\left\|\left(I-J_{r}^{A}\right)(I-r B) x-\left(I-J_{r}^{A}\right)(I-r B) y\right\|\right),
\end{aligned}
$$

where $T_{r}=J_{r}^{A}(I-r B)=(I+r A)^{-1}(I-r B)$.

We will adopt the following notation in this paper:

$$
x_{n} \rightarrow x \text { means that } x_{n} \rightarrow x \text { strongly. }
$$

\section{Main Results}

We first prove the following lemma in a real Banach space with Fréchet differentiable norm.

Lemma 11. Let $E$ be a real Banach space with Fréchet differentiable norm. Let $A: E \rightarrow 2^{E}$ be an $m$-accretive operator and let $B: E \rightarrow E$ be an $\alpha$-inverse strongly accretive mapping. Assume that $(A+B)^{-1}(0) \neq \emptyset$. Let $f: E \rightarrow E$ be a fixed contraction with coefficient $k \in(0,1)$. Let $\left\{r_{n}\right\}_{n=1}^{\infty}$ be a sequence of positive real numbers and suppose that $\left\{\alpha_{n}\right\}_{n=1}^{\infty}$ and $\left\{\beta_{n}\right\}_{n=1}^{\infty}$ are sequences in $(0,1)$. Let $\left\{x_{n}\right\}_{n=1}^{\infty} \subset E$ be a sequence generated by $x_{1} \in E$,

$$
\begin{aligned}
y_{n} & =\alpha_{n} f\left(x_{n}\right)+\left(1-\alpha_{n}\right) x_{n}, \\
x_{n+1} & =\beta_{n} x_{n}+\left(1-\beta_{n}\right) J_{r_{n}}^{A}\left(y_{n}-r_{n} B y_{n}\right),
\end{aligned}
$$

$$
n \geq 1
$$

where $J_{r_{n}}^{A}=\left(I+r_{n} A\right)^{-1}, 0<r_{n}<2 \alpha / c, n \geq 1$. Then $\left\{x_{n}\right\}_{n=1}^{\infty}$ is bounded.
Proof. For each $n \geq 1$, let $T_{n}:=J_{r_{n}}^{A}\left(I-r_{n} B\right)$ and $x^{*} \in(A+$ $B)^{-1}(0)$. Then, for all $x, y \in E$, we have

$$
\begin{aligned}
\left\|T_{n} x-T_{n} y\right\|^{2} \leq & \left\|J_{r_{n}}^{A}\left(x-r_{n} B x\right)-J_{r_{n}}^{A}\left(y-r_{n} B y\right)\right\|^{2} \\
\leq & \left\|x-y-r_{n}(B x-B y)\right\|^{2} \\
\leq & \|x-y\|^{2}-2 r_{n}\langle B x-B y, j(x-y)\rangle \\
& \quad+c r_{n}^{2}\|B x-B y\|^{2} \\
\leq & \|x-y\|^{2}-2 r_{n} \alpha\|B x-B y\|^{2} \\
& +c r_{n}^{2}\|B x-B y\|^{2} \\
= & \|x-y\|^{2}-\left(2 \alpha-c r_{n}\right) r_{n}\|B x-B y\|^{2} \\
\leq & \|x-y\|^{2} .
\end{aligned}
$$

Thus, $T_{n}$ is nonexpansive for all $n \geq 1$. Furthermore,

$$
\begin{aligned}
x & =T_{n} x \Longleftrightarrow \\
x & =\left(I+r_{n} A\right)^{-1}\left(x-r_{n} B x\right) \Longleftrightarrow \\
x-r_{n} B x & \in x+r_{n} A x \Longleftrightarrow \\
0 & \in A x+B x .
\end{aligned}
$$

Thus, $F\left(T_{n}\right)=(A+B)^{-1}(0), \forall n \geq 1$. So,

$$
\begin{aligned}
\left\|y_{n}-x^{*}\right\| \leq & \alpha_{n}\left\|f\left(x_{n}\right)-x^{*}\right\|+\left(1-\alpha_{n}\right)\left\|x_{n}-x^{*}\right\| \\
\leq & \alpha_{n} k\left\|x_{n}-x^{*}\right\|+\alpha_{n}\left\|f\left(x^{*}\right)-x^{*}\right\| \\
& +\left(1-\alpha_{n}\right)\left\|x_{n}-x^{*}\right\| \\
= & \left(1-\alpha_{n}(1-k)\right)\left\|x_{n}-x^{*}\right\| \\
& +\alpha_{n}\left\|f\left(x^{*}\right)-x^{*}\right\| .
\end{aligned}
$$

Now, using (29), we have

$$
\begin{aligned}
\left\|x_{n+1}-x^{*}\right\| \leq & \beta_{n}\left\|x_{n}-x^{*}\right\|+\left(1-\beta_{n}\right)\left\|T_{n} y_{n}-x^{*}\right\| \\
\leq & \beta_{n}\left\|x_{n}-x^{*}\right\|+\left(1-\beta_{n}\right)\left\|y_{n}-x^{*}\right\| \\
\leq & \beta_{n}\left\|x_{n}-x^{*}\right\| \\
& +\left(1-\alpha_{n}(1-k)\right)\left(1-\beta_{n}\right)\left\|x_{n}-x^{*}\right\| \\
& +\left(1-\beta_{n}\right) \alpha_{n}\left\|f\left(x^{*}\right)-x^{*}\right\| \\
= & \left(1-(1-k) \alpha_{n}\left(1-\beta_{n}\right)\right)\left\|x_{n}-x^{*}\right\| \\
& +\alpha_{n}\left(1-\beta_{n}\right)\left\|f\left(x^{*}\right)-x^{*}\right\| \\
\leq & \max \left\{\left\|x_{n}-x^{*}\right\|, \frac{\left\|f\left(x^{*}\right)-x^{*}\right\|}{1-k}\right\} \\
\leq & \cdots
\end{aligned}
$$




$$
\leq \max \left\{\left\|x_{1}-x^{*}\right\|, \frac{\left\|f\left(x^{*}\right)-x^{*}\right\|}{1-k}\right\}
$$

$<\infty$.

Hence, $\left\{x_{n}\right\}_{n=1}^{\infty}$ is bounded.

Using the idea of the proof partly taken from $[18,26$, $30,31]$, we state and prove the following strong convergence theorem.

Theorem 12. Let $E$ be a real uniformly convex Banach space which is also uniformly smooth. Let $A: E \rightarrow 2^{E}$ be an maccretive operator and let $B: E \rightarrow E$ be an $\alpha$-inverse strongly accretive. Assume that $(A+B)^{-1}(0) \neq \emptyset$. Let $f: E \rightarrow E$ be a fixed contraction with coefficient $k \in(0,1)$. Let $\left\{r_{n}\right\}_{n=1}^{\infty}$ be a sequence of positive real numbers and suppose that $\left\{\alpha_{n}\right\}_{n=1}^{\infty}$ and $\left\{\beta_{n}\right\}_{n=1}^{\infty}$ are sequences in $(0,1)$ satisfying the following conditions:

(i) $\lim _{n \rightarrow \infty} \alpha_{n}=0$;

(ii) $\sum_{n=1}^{\infty} \alpha_{n}=\infty$;

(iii) $0<\lim \inf _{n \rightarrow \infty} r_{n} \leq \lim \sup _{n \rightarrow \infty} r_{n}<2 \alpha / c$;

(iv) $0<\lim \inf _{n \rightarrow \infty} \beta_{n} \leq \lim \sup _{n \rightarrow \infty} \beta_{n}<1$.

Let the sequence $\left\{x_{n}\right\}_{n=1}^{\infty}$ be generated by (26). Then $\left\{x_{n}\right\}_{n=1}^{\infty}$ converges strongly to $z=Q_{(A+B)^{-1}(0)} f(z)$, where $Q_{(A+B)^{-1}(0)}$ is the unique sunny nonexpansive retraction of $E$ onto $(A+$ $B)^{-1}(0)$; that is, $z$ solves the variational inequality

$$
\langle(I-f) z, j(z-x)\rangle \leq 0, \quad \forall x \in(A+B)^{-1}(0) .
$$

Proof. Let $z=Q_{(A+B)^{-1}(0)} f(z)$. Then, using Lemma 6 in (26), we have that

$$
\begin{aligned}
& \left\|y_{n}-z\right\|^{2}=\left\|\alpha_{n}\left(f\left(x_{n}\right)-z\right)+\left(1-\alpha_{n}\right)\left(x_{n}-z\right)\right\|^{2} \\
& \quad=\| \alpha_{n}\left(f\left(x_{n}\right)-f(z)\right)+\left(1-\alpha_{n}\right)\left(x_{n}-z\right) \\
& +\alpha_{n}(f(z)-z)\left\|^{2} \leq\right\| \alpha_{n}\left(f\left(x_{n}\right)-f(z)\right) \\
& +\left(1-\alpha_{n}\right)\left(x_{n}-z\right) \|^{2}+2 \alpha_{n}\left\langle f(z)-z, j\left(y_{n}-z\right)\right\rangle \\
& \quad \leq \alpha_{n}\left\|f\left(x_{n}\right)-f(z)\right\|^{2}+\left(1-\alpha_{n}\right)\left\|x_{n}-z\right\|^{2} \\
& +2 \alpha_{n}\left\langle f(z)-z, j\left(y_{n}-z\right)\right\rangle \leq \alpha_{n} k^{2}\left\|x_{n}-z\right\|^{2} \\
& \quad+\left(1-\alpha_{n}\right)\left\|x_{n}-z\right\|^{2}+2 \alpha_{n}\left\langle f(z)-z, j\left(y_{n}-z\right)\right\rangle \\
& \quad=\left(1-\alpha_{n}\left(1-k^{2}\right)\right)\left\|x_{n}-z\right\|^{2}+2 \alpha_{n}\langle f(z) \\
& \left.\quad-z, j\left(y_{n}-z\right)\right\rangle .
\end{aligned}
$$

It follows from (32) and Lemma 10 that there exists a continuous, strictly increasing, and convex function $\varphi: \mathbb{R}^{+} \rightarrow \mathbb{R}^{+}$ with $\varphi(0)=0$ such that

$$
\begin{gathered}
\left\|T_{n} y_{n}-z\right\|^{2}=\left\|T_{r_{n}} y_{n}-z\right\|^{2} \leq\left\|y_{n}-z\right\|^{2}-r_{n}(2 \alpha \\
\left.-r_{n} c\right)\left\|B y_{n}-B z\right\|^{2}-\varphi\left(\|\left(I-J_{r_{n}}^{A}\right)\left(I-r_{n} B\right) y_{n}\right. \\
\left.-\left(I-J_{r_{n}}^{A}\right)\left(I-r_{n} B\right) z \|\right) \leq\left(1-\alpha_{n}\left(1-k^{2}\right)\right) \| x_{n} \\
-z \|^{2}+2 \alpha_{n}\left\langle f(z)-z, j\left(y_{n}-z\right)\right\rangle-r_{n}\left(2 \alpha-r_{n} c\right) \\
\cdot\left\|B y_{n}-B z\right\|^{2}-\varphi\left(\|\left(I-J_{r_{n}}^{A}\right)\left(I-r_{n} B\right) y_{n}\right. \\
\left.-\left(I-J_{r_{n}}^{A}\right)\left(I-r_{n} B\right) z \|\right) .
\end{gathered}
$$

Now, using (26), we have

$$
\begin{aligned}
& \left\|x_{n+1}-z\right\|^{2} \leq \beta_{n}\left\|x_{n}-z\right\|^{2}+\left(1-\beta_{n}\right)\left\|T_{n} y_{n}-z\right\|^{2} \\
& \quad \leq \beta_{n}\left\|x_{n}-z\right\|^{2}+\left(1-\beta_{n}\right)\left(1-\alpha_{n}\left(1-k^{2}\right)\right) \| x_{n} \\
& -z \|^{2}+2 \alpha_{n}\left(1-\beta_{n}\right)\left\langle f(z)-z, j\left(y_{n}-z\right)\right\rangle \\
& -r_{n}\left(2 \alpha-r_{n} c\right)\left(1-\beta_{n}\right)\left\|B y_{n}-B z\right\|^{2}-\left(1-\beta_{n}\right) \\
& \cdot \varphi\left(\|\left(I-J_{r_{n}}^{A}\right)\left(I-r_{n} B\right) y_{n}\right. \\
& \left.-\left(I-J_{r_{n}}^{A}\right)\left(I-r_{n} B\right) z \|\right)+2 \alpha_{n}\left(1-\beta_{n}\right)\langle f(z)-z, \\
& \left.\quad j\left(y_{n}-z\right)\right\rangle .
\end{aligned}
$$

Let $s_{n}=\left\|x_{n}-z\right\|^{2}, c_{n}=r_{n}\left(2 \alpha-r_{n} c\right)\left(1-\beta_{n}\right)\left\|B y_{n}-B z\right\|^{2}+(1-$ $\left.\beta_{n}\right) \varphi\left(\left\|y_{n}-r_{n} B y_{n}-T_{n} y_{n}+r_{n} B z\right\|\right)$, and $\epsilon_{n}=2\left\langle f(z)-z, j\left(y_{n}-\right.\right.$ $z)\rangle$. Then, (34) becomes

$$
\begin{aligned}
s_{n+1} \leq & \left(1-\alpha_{n}\left(1-\beta_{n}\right)\left(1-k^{2}\right)\right) s_{n}+\alpha_{n}\left(1-\beta_{n}\right) \epsilon_{n} \\
& -c_{n}, \quad n \geq 1 .
\end{aligned}
$$

We now show that $s_{n} \rightarrow 0, n \rightarrow \infty$ by considering two possible cases.

Case 1. Suppose that $\left\{s_{n}\right\}_{n=1}^{\infty}$ is eventually decreasing. That is, there exists $N \geq 0$ such that $\left\{s_{n}\right\}_{n=1}^{\infty}$ is decreasing for $n \geq N$. This implies that $\left\{s_{n}\right\}_{n=1}^{\infty}$ is convergent. It then follows from (35) that

$$
\begin{aligned}
0 \leq c_{n} \leq s_{n}-s_{n+1}+\alpha_{n}\left(1-\beta_{n}\right)\left(\epsilon_{n}-\left(1-k^{2}\right) s_{n}\right), & \\
n & \geq 1 .
\end{aligned}
$$

This implies that

$$
\begin{aligned}
& \lim _{n \rightarrow \infty}\left(\left\|B y_{n}-B z\right\|^{2}+\left\|y_{n}-r_{n} B y_{n}-T_{n} y_{n}+r_{n} B z\right\|\right) \\
& \quad=0 .
\end{aligned}
$$

Consequently,

$$
\lim _{n \rightarrow \infty}\left\|T_{n} y_{n}-y_{n}\right\|=0
$$


Since $\lim \inf _{n \rightarrow \infty} r_{n}>0$, there exists $\epsilon>0$ such that $r_{n} \geq \epsilon$, $\forall n \geq 1$. Then, by Lemma 7, we have

$$
\lim _{n \rightarrow \infty}\left\|T_{\epsilon} y_{n}-y_{n}\right\| \leq 2 \lim _{n \rightarrow \infty}\left\|T_{n} y_{n}-y_{n}\right\|=0 .
$$

Also, from (26), we obtain

$$
\left\|y_{n}-x_{n}\right\|=\alpha_{n}\left\|f\left(x_{n}\right)-x_{n}\right\| \longrightarrow 0, \quad n \longrightarrow \infty .
$$

Since $F\left(T_{\epsilon}\right)=(A+B)^{-1}(0)$, it follows from Lemma 5 that

$$
\limsup _{n \rightarrow \infty}\left\langle f(z)-z, j\left(y_{n}-z\right)\right\rangle \leq 0 .
$$

Equivalently, $\lim \sup _{n \rightarrow \infty} \epsilon_{n} \leq 0$. We observe further that

$$
\begin{aligned}
\left\|x_{n}-T_{n} y_{n}\right\| \leq\left\|y_{n}-x_{n}\right\|+\left\|T_{n} y_{n}-y_{n}\right\| \longrightarrow & 0, \\
& n \longrightarrow \infty .
\end{aligned}
$$

Again from (26), we have that

$$
\left\|x_{n+1}-T_{n} y_{n}\right\|=\beta_{n}\left\|x_{n}-T_{n} y_{n}\right\| \longrightarrow 0, \quad n \longrightarrow \infty .
$$

Hence,

$$
\begin{aligned}
\left\|x_{n+1}-x_{n}\right\| \leq\left\|x_{n}-T_{n} y_{n}\right\|+\left\|x_{n+1}-T_{n} y_{n}\right\| & \longrightarrow 0, \\
& n \longrightarrow \infty .
\end{aligned}
$$

From (36), we have that

$$
s_{n} \leq\left[\frac{s_{n}-s_{n+1}}{\alpha_{n}\left(1-\beta_{n}\right)}+\epsilon_{n}\right] \frac{1}{\left(1-k^{2}\right)} .
$$

Observe that $\lim \inf _{n \rightarrow \infty}\left(\left(s_{n}-s_{n+1}\right) / \alpha_{n}\left(1-\beta_{n}\right)\right)=0$ since $\sum_{n=1}^{\infty} \alpha_{n}\left(1-\beta_{n}\right)=\infty$. Taking lim inf in (45), we have

$$
\begin{aligned}
& \lim _{n \rightarrow \infty} s_{n} \leq \frac{1}{\left(1-k^{2}\right)}\left[\liminf _{n \rightarrow \infty}\left[\frac{s_{n}-s_{n+1}}{\alpha_{n}\left(1-\beta_{n}\right)}+\epsilon_{n}\right]\right] \\
& \leq \frac{1}{\left(1-k^{2}\right)}\left[\liminf _{n \rightarrow \infty}\left[\frac{s_{n}-s_{n+1}}{\alpha_{n}\left(1-\beta_{n}\right)}\right]+\limsup _{n \rightarrow \infty} \epsilon_{n}\right] \\
& \quad \leq 0
\end{aligned}
$$

and thus $\left\{s_{n}\right\}_{n=1}^{\infty}$ must converge to zero.

Case 2. Suppose $\left\{s_{n}\right\}_{n=1}^{\infty}$ is not eventually decreasing. Hence, we can find a subsequence $\left\{s_{n_{k}}\right\}_{k=1}^{\infty}$ of $\left\{s_{n}\right\}_{n=1}^{\infty}$ such that $s_{n_{k}} \leq$ $s_{n_{k}+1}, \forall k \geq 1$. Then we can define a subsequence $\left\{S_{\tau(n)}\right\}$ as in Lemma 3 so that

$$
\max \left\{s_{\tau(n)}, s_{n}\right\} \leq s_{\tau(n)+1}, \quad \forall n>n_{0} .
$$

This implies from (36) that

$$
\begin{aligned}
0 & \leq c_{\tau(n)} \leq \alpha_{\tau(n)}\left(1-\beta_{\tau(n)}\right)\left(\epsilon_{\tau(n)}-\left(1-k^{2}\right) s_{\tau(n)}\right) \\
& \longrightarrow 0, \quad n \longrightarrow \infty
\end{aligned}
$$

and thus $\left\|T_{\tau(n)} y_{\tau(n)}-y_{\tau(n)}\right\| \rightarrow 0, n \rightarrow \infty$. In a similar way to Case 1, we have

$$
\limsup _{n \rightarrow \infty}\left\langle f(z)-z, j\left(y_{\tau(n)}-z\right)\right\rangle \leq 0 .
$$

Equivalently, $\lim \sup _{n \rightarrow \infty} \epsilon_{\tau(n)} \leq 0$ which by (48) implies that $\lim \sup _{n \rightarrow \infty} s_{\tau(n)} \leq 0$. By repeating the same arguments in Case 1 , we can show that

$$
\left\|y_{n}-x_{n}\right\|=\alpha_{n}\left\|f\left(x_{n}\right)-x_{n}\right\| \longrightarrow 0,
$$$$
n \longrightarrow \infty
$$

$$
\left\|x_{\tau(n)+1}-x_{\tau(n)}\right\| \longrightarrow 0, \quad n \longrightarrow \infty .
$$

Using the definition of $\left\{s_{n}\right\}$, we have

$$
\begin{aligned}
& \left|s_{\tau(n)+1}-s_{\tau(n)}\right|=\left|\left\|x_{\tau(n)+1}-z\right\|^{2}-\left\|x_{\tau(n)}-z\right\|^{2}\right| \\
& \quad \leq\left\|x_{\tau(n)+1}-x_{\tau(n)}\right\|\left(\left\|x_{\tau(n)+1}-z\right\|+\left\|x_{\tau(n)}-z\right\|\right) \\
& \quad \longrightarrow 0, \quad n \longrightarrow \infty .
\end{aligned}
$$

This together with (47) implies that

$$
\begin{aligned}
0 \leq s_{n} \leq s_{\tau(n)+1}=s_{\tau(n)}+\left(s_{\tau(n)+1}-s_{\tau(n)}\right) \longrightarrow & 0, \\
& n \longrightarrow \infty .
\end{aligned}
$$

Consequently, we have that $s_{n} \rightarrow 0, n \rightarrow \infty$ and so $x_{n} \rightarrow z$, $n \rightarrow \infty$

If the mapping $f$ maps every point in $E$ to a fixed element, then we have the following result.

Corollary 13. Let $E$ be a real uniformly convex Banach space which is also uniformly smooth. Let $A: E \rightarrow 2^{E}$ be an $m$ accretive operator and let $B: E \rightarrow E$ be an $\alpha$-inverse strongly accretive. Assume that $(A+B)^{-1}(0) \neq \emptyset$. Let $u$ be a fixed element in $E$. Let $\left\{r_{n}\right\}_{n=1}^{\infty}$ be a sequence of positive real numbers and suppose that $\left\{\alpha_{n}\right\}_{n=1}^{\infty}$ and $\left\{\beta_{n}\right\}_{n=1}^{\infty}$ are sequences in $(0,1)$ satisfying the following conditions:

(i) $\lim _{n \rightarrow \infty} \alpha_{n}=0$;

(ii) $\sum_{n=1}^{\infty} \alpha_{n}=\infty$;

(iii) $0<\lim _{\inf _{n \rightarrow \infty}} r_{n} \leq \lim \sup _{n \rightarrow \infty} r_{n}<2 \alpha / c$;

(iv) $0<\lim \inf _{n \rightarrow \infty} \beta_{n} \leq \lim \sup _{n \rightarrow \infty} \beta_{n}<1$.

For a fixed element $u \in E$, let the sequence $\left\{x_{n}\right\}_{n=1}^{\infty}$ be generated by $x_{1} \in E$,

$$
\begin{aligned}
y_{n} & =\alpha_{n} u+\left(1-\alpha_{n}\right) x_{n}, \\
x_{n+1} & =\beta_{n} x_{n}+\left(1-\beta_{n}\right) J_{r_{n}}^{A}\left(y_{n}-r_{n} B y_{n}\right), \quad n \geq 1,
\end{aligned}
$$

where $J_{r_{n}}^{A}=\left(I+r_{n} A\right)^{-1}$. Then $\left\{x_{n}\right\}_{n=1}^{\infty}$ converges strongly to $z=Q_{(A+B)^{-1}(0)} u$, where $Q_{(A+B)^{-1}(0)}$ is the unique sunny nonexpansive retraction of $E$ onto $(A+B)^{-1}(0)$; that is, $z$ solves the variational inequality

$$
\langle z-u, j(z-x)\rangle \leq 0, \quad \forall x \in(A+B)^{-1}(0) .
$$

Recall that a mapping $h: E \rightarrow E$ is said to be weakly contractive if

$$
\|h(x)-h(y)\| \leq\|x-y\|-\psi(\|x-y\|), \quad \forall x, y \in E,
$$


where $\psi:[0, \infty) \rightarrow[0, \infty)$ is a continuous and strictly increasing function such that $\psi$ is positive on $(0, \infty)$ and $\psi(0)=0$. Let $\psi(t):=(1-k) t$ for $t \in[0, \infty)$, where $k \in$ $(0,1)$. Then we see that the weakly contractive mapping $h$ is a contraction with constant $k$. The next lemma gives existence of unique fixed point for weakly contractive mapping.

Lemma 14 (see [32, Theorem 2]). Let $(X, d)$ be a complete metric space and $h$ a weakly contractive mapping on $X$. Then, $h$ has a unique fixed point $p$ in $X$. Moreover, for $x \in X,\left\{h^{n} x\right\}$ converges strongly to $p$.

The following lemma will be used in the next theorem.

Lemma 15 (see $[33,34])$. Let $\left\{s_{n}\right\}$ and $\left\{\gamma_{n}\right\}$ be two sequences of nonnegative real numbers and $\left\{\alpha_{n}\right\}$ a sequence of positive numbers satisfying the following conditions:

(i) $\sum_{n=1}^{\infty} \alpha_{n}=\infty$;

(ii) $\lim _{n \rightarrow \infty}\left(\gamma_{n} / \alpha_{n}\right)=0$.

Let the recursive inequality

$$
s_{n+1} \leq s_{n}-\alpha_{n} \psi\left(s_{n}\right)+\gamma_{n}, \quad n \geq 1,
$$

where $\psi:[0, \infty) \rightarrow[0, \infty)$ is a continuous and strictly increasing function such that $\psi$ is positive on $(0, \infty)$ and $\psi(0)=$ 0 . Then, $\lim _{n \rightarrow \infty} s_{n}=0$.

We now consider the viscosity approximation method with weakly contractive mapping in the following theorem.

Theorem 16. Let $E$ be a real uniformly convex Banach space which is also uniformly smooth. Let $A: E \rightarrow 2^{E}$ be an maccretive operator and let $B: E \rightarrow E$ be an $\alpha$-inverse strongly accretive. Assume that $(A+B)^{-1}(0) \neq \emptyset$. Let $h: E \rightarrow E$ be a weakly contractive mapping. Let $\left\{r_{n}\right\}_{n=1}^{\infty}$ be a sequence of positive real numbers and suppose that $\left\{\alpha_{n}\right\}_{n=1}^{\infty}$ and $\left\{\beta_{n}\right\}_{n=1}^{\infty}$ are sequences in $(0,1)$ satisfying the following conditions:

(i) $\lim _{n \rightarrow \infty} \alpha_{n}=0$;

(ii) $\sum_{n=1}^{\infty} \alpha_{n}=\infty$;

(iii) $0<\lim \inf _{n \rightarrow \infty} r_{n} \leq \lim \sup _{n \rightarrow \infty} r_{n}<2 \alpha / c$;

(iv) $0<\lim _{\inf _{n \rightarrow \infty}} \beta_{n} \leq \lim \sup _{n \rightarrow \infty} \beta_{n}<1$.

Let $\left\{x_{n}\right\}_{n=1}^{\infty} \subset E$ be a sequence generated by $x_{1} \in E$,

$$
\begin{aligned}
y_{n} & =\alpha_{n} h\left(x_{n}\right)+\left(1-\alpha_{n}\right) x_{n}, \\
x_{n+1} & =\beta_{n} x_{n}+\left(1-\beta_{n}\right) J_{r_{n}}^{A}\left(y_{n}-r_{n} B y_{n}\right), \quad n \geq 1 .
\end{aligned}
$$

Then $\left\{x_{n}\right\}_{n=1}^{\infty}$ converges strongly to $z=Q_{(A+B)^{-1}(0)} h(z)$, where $Q_{(A+B)^{-1}(0)}$ is the unique sunny nonexpansive retraction of $E$ onto $(A+B)^{-1}(0)$; that is, $z$ solves the variational inequality

$$
\langle(I-h) z, j(z-x)\rangle \leq 0, \quad \forall x \in(A+B)^{-1}(0) .
$$

Proof. We first observe that $Q_{(A+B)^{-1}(0)} h$ is a weakly contractive mapping. Indeed, $\forall x, y \in E$,

$$
\begin{aligned}
& \left\|Q_{(A+B)^{-1}(0)} h(x)-Q_{(A+B)^{-1}(0)} h(y)\right\| \leq\|h(x)-h(y)\| \\
& \quad \leq\|x-y\|-\psi(\|x-y\|) .
\end{aligned}
$$

Then Lemma 14 assures that there exists a unique element $z \epsilon$ $(A+B)^{-1}(0)$ such that $z=Q_{(A+B)^{-1}(0)} h(z)$. Now, define an iterative scheme as follows:

$$
\begin{aligned}
& w_{n+1} \\
& \quad=\beta_{n} w_{n} \\
& +\left(1-\beta_{n}\right) J_{r_{n}}^{A}\left(I-r_{n} B\right)\left[\alpha_{n} h(z)+\left(1-\alpha_{n}\right) w_{n}\right], \\
& n \geq 1 .
\end{aligned}
$$

Let $\left\{w_{n}\right\}$ be the sequence generated by (60). Then, Corollary 13 with $f=h(z)$ a constant assures that $\left\{w_{n}\right\}$ converges strongly to $z=Q_{(A+B)^{-1}(0)} h(z)$. Now, for $n \geq 1$, we obtain

$$
\begin{aligned}
& \left\|x_{n+1}-w_{n+1}\right\|=\| \beta_{n} x_{n} \\
& \quad+\left(1-\beta_{n}\right) J_{r_{n}}^{A}\left(I-r_{n} B\right)\left[\alpha_{n} h\left(x_{n}\right)+\left(1-\alpha_{n}\right) x_{n}\right] \\
& \quad-\beta_{n} w_{n} \\
& \quad-\left(1-\beta_{n}\right) J_{r_{n}}^{A}\left(I-r_{n} B\right)\left[\alpha_{n} h(z)+\left(1-\alpha_{n}\right) w_{n}\right] \| \\
& \quad \leq \beta_{n}\left\|x_{n}-w_{n}\right\|+\left(1-\beta_{n}\right) \\
& \quad-\|\left[\alpha_{n} h\left(x_{n}\right)+\left(1-\alpha_{n}\right) x_{n}\right] \\
& -\left[\alpha_{n} h(z)+\left(1-\alpha_{n}\right) w_{n}\right]\left\|\leq \beta_{n}\right\| x_{n}-w_{n} \|+\alpha_{n}(1 \\
& \left.-\beta_{n}\right)\left\|h\left(x_{n}\right)-h(z)\right\|+\left(1-\alpha_{n}\right)\left(1-\beta_{n}\right)\left\|x_{n}-w_{n}\right\| \\
& \quad \leq \beta_{n}\left\|x_{n}-w_{n}\right\|+\alpha_{n}\left(1-\beta_{n}\right)\left[\left\|h\left(x_{n}\right)-h\left(w_{n}\right)\right\|\right. \\
& \left.\quad+\left\|h\left(w_{n}\right)-h(z)\right\|\right]+\left(1-\alpha_{n}\right)\left(1-\beta_{n}\right)\left\|x_{n}-w_{n}\right\| \\
& \quad \leq \beta_{n}\left\|x_{n}-w_{n}\right\|+\alpha_{n}\left(1-\beta_{n}\right)\left[\left\|x_{n}-w_{n}\right\|\right. \\
& \left.-\psi\left(\left\|x_{n}-w_{n}\right\|\right)\right]+\alpha_{n}\left(1-\beta_{n}\right)\left[\left\|w_{n}-z\right\|\right. \\
& \left.-\psi\left(\left\|w_{n}-z\right\|\right)\right]+\left(1-\alpha_{n}\right)\left(1-\beta_{n}\right)\left\|x_{n}-w_{n}\right\| \\
& \quad=\left\|x_{n}-w_{n}\right\|-\alpha_{n}\left(1-\beta_{n}\right) \psi\left(\left\|x_{n}-w_{n}\right\|\right)+\alpha_{n}(1 \\
& \left.-\beta_{n}\right)\left[\left\|w_{n}-z\right\|-\psi\left(\left\|w_{n}-z\right\|\right)\right] \leq\left\|x_{n}-w_{n}\right\| \\
& -\alpha_{n}\left(1-\beta_{n}\right) \psi\left(\left\|x_{n}-w_{n}\right\|\right)+\alpha_{n}\left(1-\beta_{n}\right)\left\|w_{n}-z\right\| .
\end{aligned}
$$

Take $s_{n}=\left\|x_{n}-w_{n}\right\|$; we have the following recursive inequality:

$$
s_{n+1} \leq s_{n}-\alpha_{n} \psi\left(s_{n}\right)+\alpha_{n}\left\|w_{n}-z\right\| .
$$

Since $\left\|w_{n}-z\right\| \rightarrow 0, n \rightarrow \infty$, it follows from Lemma 15 that $\left\|x_{n}-w_{n}\right\| \rightarrow 0, n \rightarrow \infty$. Furthermore,

$$
\lim _{n \rightarrow \infty}\left\|x_{n}-z\right\| \leq \lim _{n \rightarrow \infty}\left(\left\|x_{n}-w_{n}\right\|+\left\|w_{n}-z\right\|\right)=0 .
$$

This completes the proof.

Remark 17. All the results in this paper can still be obtained if error terms are added to our iterative scheme (26). Thus, addition of error terms to our iterative scheme (26) leads to no further generalization of the results obtained in this paper. 
Remark 18. We make the following comments which highlight our contributions in this paper (please see also [31]):

(1) The results of Wei and Shi [23] do not hold in $L_{p}$ spaces with $p \neq 2$. Here in this paper, our results hold, in particular, in $L_{p}$ spaces with $p \geq 2$. Furthermore, the condition $\sum_{n=1}^{\infty}\left|r_{n+1}-r_{n}\right|<\infty$ assumed in the results of Wei and Shi [23] is dispensed with in our results. Thus, our results improve on the results of Wei and Shi [23].

(2) The condition $\sum_{n=1}^{\infty}\left|r_{n+1}-r_{n}\right|<\infty$ assumed in the results of Wei and Duan [25] is dispensed with in our results. Also, the condition

$$
\langle B x-B y, J((I-r B) x-(I-r B)) y\rangle \geq 0,
$$

for all $x, y \in E$ and for all $r>0$ assumed in the results of Wei and Duan [25], is dispensed with in our results. Hence, our results improved on the results of Wei and Duan [25].

(3) Our results extend the results of [21-23, 25] from q-uniformly smooth Banach spaces to uniformly smooth Banach spaces. By Remark 1, our results also extend the results of $[21-23,25]$ from the class of inverse strongly accretive operators of order $q$ to the more general class of inverse strongly accretive operators.

(4) In our result in this paper, we consider the viscosity approximation method with weakly contractive mapping for solving (10), which extends all the previous results concerning viscosity approximation method with fixed contraction for solving (10) in $q$-uniformly smooth Banach spaces to uniformly smooth Banach spaces. Our result in this paper also complements the results of Shehu and Cai [31].

Remark 19. We specifically clarify the differences between the results of this paper and the results obtained in $[23,25]$ :

(1) We state here that the results obtained in Wei and Duan [25] and Wei and Shi [23] do not hold in $L_{p}$ spaces with $p \neq 2$ because the duality mapping $J$ is assumed to be weakly sequentially continuous but here in this paper, our results are applicable, in particular, in $L_{p}$ spaces with $2 \leq p<\infty$ because the weak sequential continuity of the duality mapping $J$ is dispensed with.

(2) The choice of sequence $\left\{r_{n}\right\}$ of positive real numbers with

$$
r_{n}= \begin{cases}n^{-1 / 2}, & \text { if } n \text { is odd } \\ \left(n^{-1 / 2}-1\right)^{-1}, & \text { if } n \text { is even }\end{cases}
$$

does not satisfy the extra condition $\sum_{n=1}^{\infty}\left|r_{n+1}-r_{n}\right|<$ $\infty$ assumed in Wei and Duan [25] and Wei and Shi [23].

\section{Conflict of Interests}

The author declares that there is no conflict of interests regarding the publication of this paper.

\section{References}

[1] Z.-B. Xu and G. F. Roach, "Characteristic inequalities of uniformly convex and uniformly smooth Banach spaces," Journal of Mathematical Analysis and Applications, vol. 157, no. 1, pp. 189210, 1991.

[2] W. Takahashi, Nonlinear Functional Analysis-Fixed Point Theory and Applications, Yokohama Publishers, Yokohama, Japan, 2000.

[3] W. Takahashi, "Proximal point algorithms and four resolvents of nonlinear operators of monotone type in Banach spaces," Taiwanese Journal of Mathematics, vol. 12, no. 8, pp. 1883-1910, 2008.

[4] S. Reich, "Strong convergence theorems for resolvents of accretive operators in Banach spaces," Journal of Mathematical Analysis and Applications, vol. 75, no. 1, pp. 287-292, 1980.

[5] T. Kato, "Nonlinear semigroups and evolution equations," Journal of the Mathematical Society of Japan, vol. 19, no. 4, pp. 508-520, 1967.

[6] P. L. Combettes and V. R. Wajs, "Signal recovery by proximal forward-backward splitting," Multiscale Modeling and Simulation, vol. 4, no. 4, pp. 1168-1200, 2005.

[7] P. L. Lions and B. Mercier, "Splitting algorithms for the sum of two nonlinear operators," SIAM Journal on Numerical Analysis, vol. 16, no. 6, pp. 964-979, 1979.

[8] G. B. Passty, "Ergodic convergence to a zero of the sum of monotone operators in Hilbert space," Journal of Mathematical Analysis and Applications, vol. 72, no. 2, pp. 383-390, 1979.

[9] P. Tseng, "Modified forward-backward splitting method for maximal monotone mappings," SIAM Journal on Control and Optimization, vol. 38, no. 2, pp. 431-446, 2000.

[10] H. Brezis and P. L. Lions, "Produits infinis de resolvantes," Israel Journal of Mathematics, vol. 29, no. 4, pp. 329-345, 1978.

[11] G. H.-G. Chen and R. T. Rockafellar, "Convergence rates in forward-backward splitting," SIAM Journal on Optimization, vol. 7, no. 2, pp. 421-444, 1997.

[12] O. Güeler, "On the convergence of the proximal point algorithm for convex minimization," SIAM Journal on Control and Optimization, vol. 29, no. 2, pp. 403-419, 1991.

[13] B. Martinet, "Régularisation d'inéquations variationnelles par approximations successives," Revue Française d'Automatique, Informatique, Recherche Opérationnelle, vol. 4, no. 3, pp. 154158, 1970.

[14] R. T. Rockafellar, "Monotone operators and the proximal point algorithm," SIAM Journal on Control and Optimization, vol. 14, no. 5, pp. 877-898, 1976.

[15] J. C. Dunn, "Convexity, monotonicity, and gradient processes in Hilbert space," Journal of Mathematical Analysis and Applications, vol. 53, no. 1, pp. 145-158, 1976.

[16] P. L. Combettes, "Iterative construction of the resolvent of a sum of maximal monotone operators," Journal of Convex Analysis, vol. 16, no. 3-4, pp. 727-748, 2009.

[17] W. Takahashi, N.-C. Wong, and J.-C. Yao, “Two generalized strong convergence theorems of Halpern's type in Hilbert spaces and applications," Taiwanese Journal of Mathematics, vol. 16, no. 3, pp. 1151-1172, 2012. 
[18] F. Wang and H. Cui, "On the contraction-proximal point algorithms with multi-parameters," Journal of Global Optimization, vol. 54, no. 3, pp. 485-491, 2012.

[19] H. Zegeye and N. Shahzad, "Strong convergence theorems for a common zero of a finite family of m-accretive mappings," Nonlinear Analysis, Theory, Methods and Applications, vol. 66, no. 5, pp. 1161-1169, 2007.

[20] H. Zegeye and N. Shahzad, "Solutions of variational inequality problems in the set of fixed points of pseudocontractive mappings," Carpathian Journal of Mathematics, vol. 30, pp. 257-265, 2014.

[21] G. López, V. Martín-Márquez, F. Wang, and H.-K. Xu, "Forward-backward splitting methods for accretive operators in Banach spaces," Abstract and Applied Analysis, vol. 2012, Article ID 109236, 25 pages, 2012.

[22] P. Cholamjiak, "A generalized forward-backward splitting method for solving quasi inclusion problems in Banach spaces," Numerical Algorithms, 2015.

[23] L. Wei and A. Shi, "Splitting-midpoint method for zeros of the sum of accretive operator and $\mu$-inversely strongly accretive operator in a q-uniformly smooth Banach space and its applications," Journal of Inequalities and Applications, vol. 2015, article 183, 2015.

[24] V. Colao and G. Marino, "Common fixed points of strict pseudocontractions by iterative algorithms," Journal of Mathematical Analysis and Applications, vol. 382, no. 2, pp. 631-644, 2011.

[25] L. Wei and L. Duan, "A new iterative algorithm for the sum of two different types of finitely many accretive operators in Banach space and its connection with capillarity equation," Fixed Point Theory and Applications, vol. 2015, article 25, 2015.

[26] P.-E. Maingé, "Strong convergence of projected subgradient methods for nonsmooth and nonstrictly convex minimization," Set-Valued Analysis, vol. 16, no. 7-8, pp. 899-912, 2008.

[27] H.-K. Xu, "Iterative algorithms for nonlinear operators," Journal of the London Mathematical Society, vol. 66, no. 1, pp. 240-256, 2002.

[28] H.-K. Xu, "Viscosity approximation methods for nonexpansive mappings," Journal of Mathematical Analysis and Applications, vol. 298, no. 1, pp. 279-291, 2004.

[29] S. Suantai and P. Cholamjiak, "Weak convergence theorems for a countable family of strict pseudocontractions in banach spaces," Fixed Point Theory and Applications, vol. 2010, Article ID 632137, 2010.

[30] H. Cui and M. Su, "On sufficient conditions ensuring the norm convergence of an iterative sequence to zeros of accretive operators," Applied Mathematics and Computation, vol. 258, pp. 67-71, 2015.

[31] Y. Shehu and G. Cai, "Strong convergence result of forwardbackward splitting methods for accretive operators in Banach Spaces with applications," Revista de la Real Academia de Ciencias Exactas, Físicas y Naturales. Serie A. Matemáticas, In press.

[32] B. E. Rhoades, "Some theorems on weakly contractive maps," Nonlinear Analysis, Theory, Methods and Applications, vol. 47, no. 4, pp. 2683-2693, 2001.

[33] Y. I. Alber and A. N. Iusem, "Extension of subgradient techniques for nonsmooth optimization in Banach spaces," SetValued Analysis, vol. 9, no. 4, pp. 315-335, 2001.

[34] Y. Alber, S. Reich, and J.-C. Yao, "Iterative methods for solving fixed-point problems with nonself-mappings in banach spaces," Abstract and Applied Analysis, vol. 2003, no. 4, pp. 193-216, 2003. 


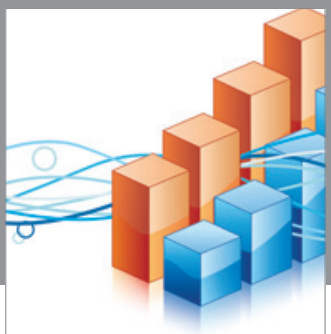

Advances in

Operations Research

vatem alat4

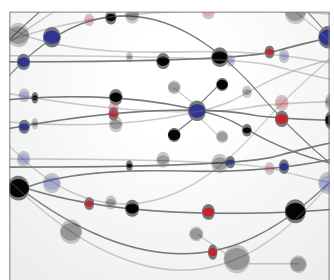

\section{The Scientific} World Journal
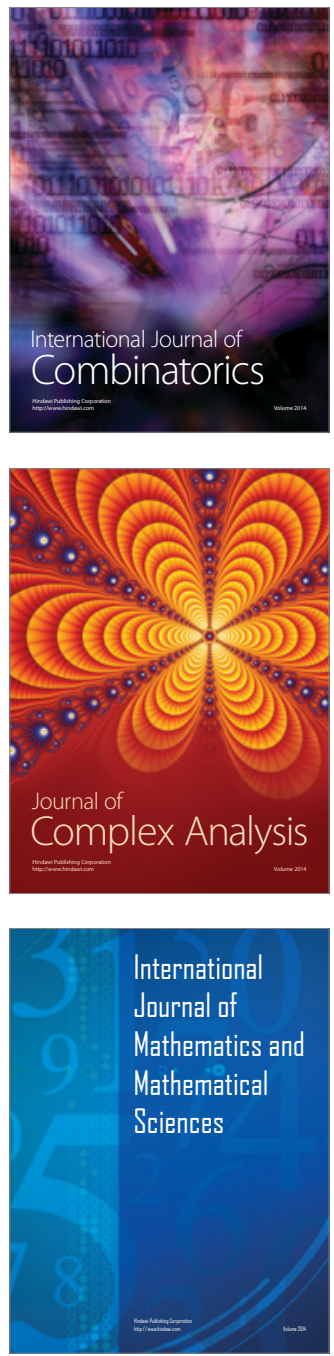
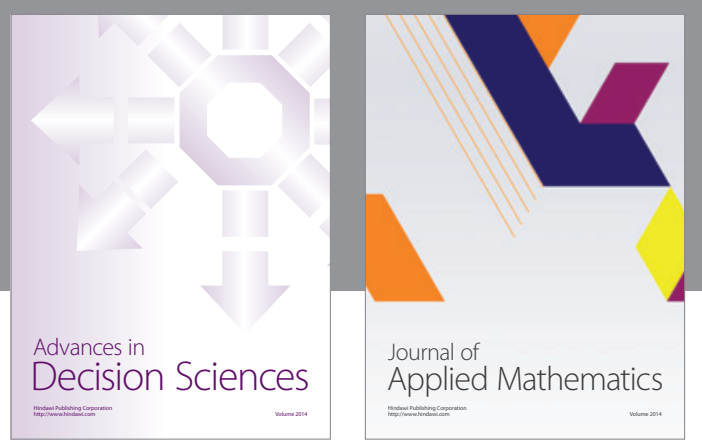

Algebra

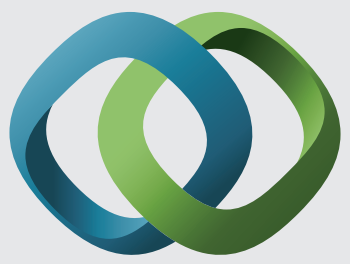

\section{Hindawi}

Submit your manuscripts at

http://www.hindawi.com
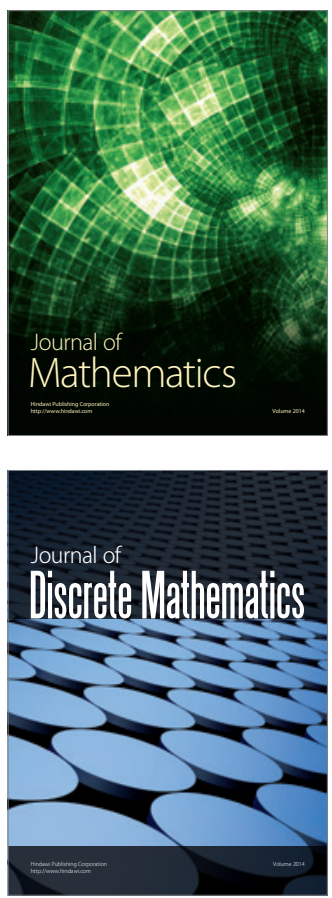

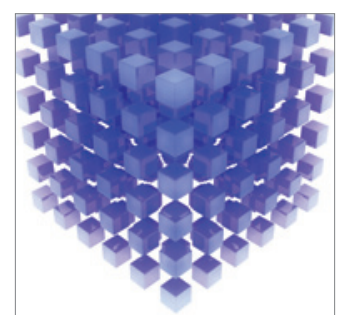

Mathematical Problems in Engineering
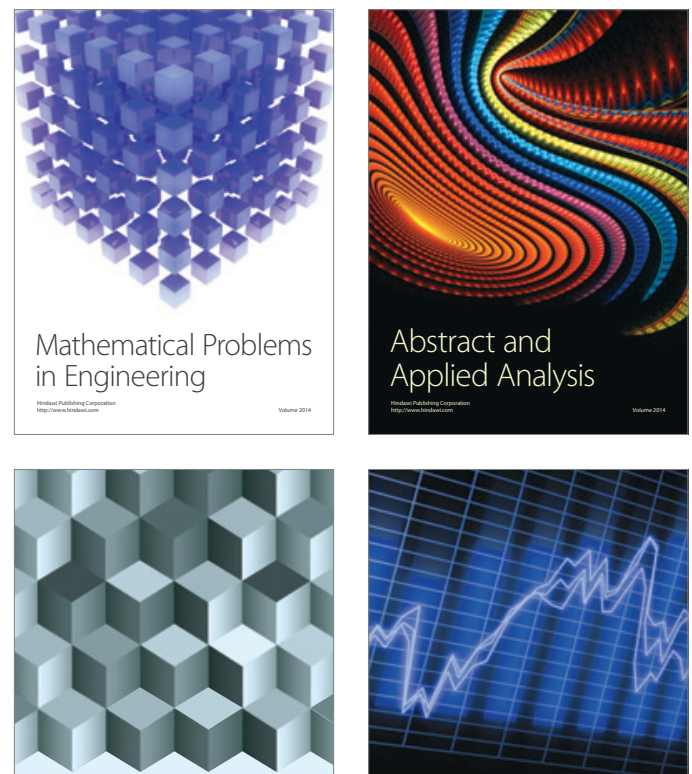

Journal of

Function Spaces

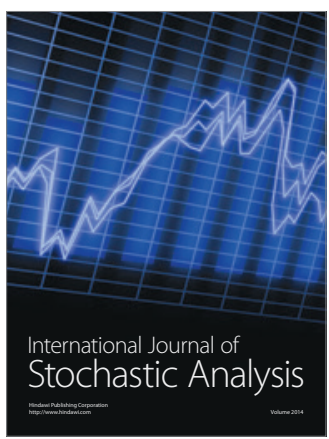

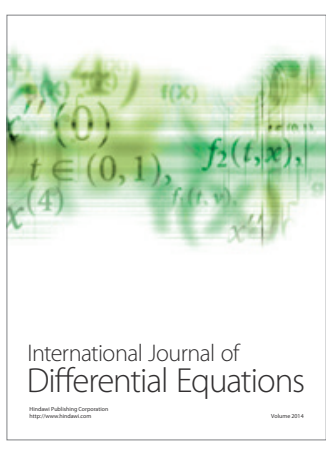
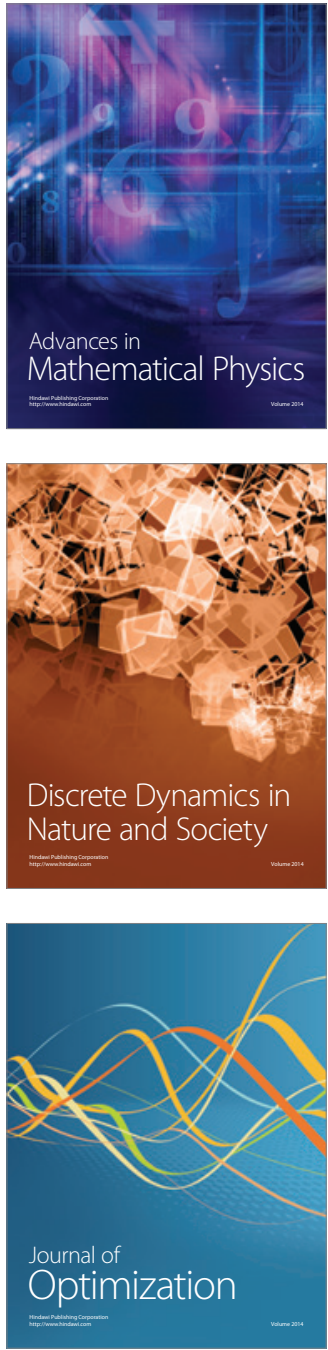